\title{
CONTABILIDAD FINANCIERA Y VALUACIÓN DE ACTIVOS INTANGIBLES
}

\author{
FINANCIAL ACCOUNTING AND VALUATION OF INTANGIBLE ASSETS \\ Raúl Alberto Arrarte Mera* \\ Docente Principal de la Facultad de Ciencias Contables \\ Universidad Nacional Mayor de San Marcos-UNMSM / Lima-Perú \\ [Recepción: Marzo de 2015 / Conformidad: Abril 2015]
}

\section{RESUMEN}

Esta investigación fue auspiciada por el Vicerrectorado de Investigación de la UNMSM, en el año 2014; es una revisión exploratoria documental que concluye en que no hay evidencias claras por parte de la Junta de Estándares Internacionales de Contabilidad o International Accounting Standards Board (IASB), para reconocer como intangibles a las herramientas fundamentales en la gestión de los negocios que utilizan las empresas integradas en el Factor Humano, el Factor Cliente y el Factor Organizacional. Que es de necesidad pública ampliar el actual Marco Conceptual para la Preparación y Presentación de Estados Financieros de las normas internacionales vigentes hacia la información no financiera; validando así, instrumentos prospectivos como la Planificación Estratégica, el Cuadro de Mando Integral, Capital Intelectual y el Valor Económico Agregado. Haciendo que la ciencia contable sea predictible en el largo plazo. Si la controversia se plantea en los términos antes esbozados, sólo quedaría promocionar el uso obligatorio de las herramientas de gestión ya citados. Realzando y normalizando así, el nivel técnico de la información no financiera a nivel global.

Palabras clave:

Activos intangibles; capital intelectual; Normas Internacionales de Información Financiera; responsabilidad social empresarial; valor económico agregado.

\begin{abstract}
This investigation was sponsored by Vice President for Research of San Marcos, in 2014. It is an exploratory documentary review which concludes that there is no clear evidence by the Accounting Standards Board (IASB) to recognize as intangible the fundamental tools used in the business management of integrated companies into the Human Factor, Customer Factor and Organizational Factor. It is of public need to expand the current Framework for the Preparation and Presentation of Financial Statements of existing international standards to non-financial information. Validating in those terms, prospective instruments like Strategic Planning, the Balanced Scorecard, Intellectual Capital and Added Economic Value; making the accounting science predictable in the long term. If a dispute is stated in the terms outlined above, it would only promote the mandatory use of management tools already mentioned; enhancing and thus normalizing, the technical level of non-financial information globally.
\end{abstract}

\section{Keywords:}

Intangible assets; intellectual capital; International Financial Reporting Standards; corporate social responsibility; added economic value.

\footnotetext{
* Doctor en Ciencias Contables y Empresariales, Contador Público - UNMSM. Maestro en Administración - USMP.

Email: rarrartem@unmsm.edu.pe
} 


\section{INTRODUCCIÓN}

La contabilidad internacional ha revolucionado la información financiera en las bolsas de valores del orbe, merced a la gestión de la Junta de Estándares Internacionales de Contabilidad (IASB), encargada de emitir las Normas Internacionales de Información Financiera (NIIF), modelo contable que será aplicado a nivel mundial, a más tardar en el año 2015.

En nuestro país (Perú), este patrón universal no ha pasado desapercibido. Así, desde octubre de 2010 la Superintendencia del Mercado de Valores (SMV), encargada del control empresarial público y privado de las sociedades anónimas abiertas ${ }^{1}$, hará de obligatorio cumplimiento la presentación de la información financiera preparada de acuerdo a las Normas Internacionales de Información Financiera (NIIF) conocidas también como IFRS (International Financial Reporting Standards), por sus siglas en inglés), al 31 de diciembre de 2014.

Este tema es de interés en muchas investigaciones alrededor del mundo, como lo expresa muy acertadamente Cañibano (2000), "La inversión en conocimiento, capital humano, innovación de nuevos productos, tecnología, redes de fidelidad con los consumidores, etc., son ejemplos de factores de naturaleza inmaterial que determinan el éxito empresarial pero que, con la regulación vigente, son excluidos de los balances empresariales debido, entre otras causas, a la "incertidumbre" existente respecto a la generación de beneficios económicos futuros”. Pero nuestra inquietud, se centra en integrar las nuevas técnicas empresariales globales de uso generalizado que califican como intangibles aún no identificados como tal, en el marco de las NIC 38 y la NIIF 6 en el campo de la contabilidad financiera.

Estamos viviendo en una era de brusca ruptura con el pasado. Ya no podemos hacer nada importante, si nos conformamos con desarrollar las cosas un poco mejor que los demás, o de una manera diferente de cómo las hacíamos anteriormente. Es necesario ampliar la relación de los activos intangibles. Nos corresponde a los investigadores colaborar en la di- fusión de la normatividad del modelo IASB. Es una oportunidad para analizar ideas nuevas relacionadas con las Normas Internacionales de Contabilidad 38 y NIIF 6, que regulan los intangibles en el mundo.

El desarrollo de este trabajo presenta la siguiente estructura: introducción, objetivos, metodología aplicada, marco teórico, enfoque, resultados, discusión, conclusiones y recomendaciones. Finalmente, exponemos el material bibliográfico utilizado para sustentar nuestro punto de vista.

\section{OBJETIVOS}

La investigación planteó desarrollar los siguientes objetivos:

a) Identificar las principales debilidades del modelo de información contable vigente respecto a la valuación de los Activos Intangibles en el marco de las Normas Internacionales de Información Financiera.

b) Analizar y evaluar la NIC 38 y la NIIF 6, como tratamiento a los Activos Intangibles para integrar las nuevas modalidades empresariales globales y mejorar la calidad de información.

c) Proponer información contable externa que facilite la valuación de los activos intangibles

\section{METODOLOGÍA, TÉCNICAS DE INVESTIGACIÓN Y MUESTRA}

Se utilizó una metodología no experimental fundamentalmente, cualitativa y teórica-exploratoria-documental, ya que los problemas y cuestiones planteados en los objetivos son aspectos dinámicos difíciles de estudiar mediante una posición estrictamente cuantitativa.

De las 234 empresas que listan en la Bolsa de Valores de Lima al 31 de diciembre de 2014, se identificaron 100 empresas industriales de diferentes sectores elegidos por su volumen de ventas y por el nivel de negocios internacionales, cuya composición de la muestra es la siguiente:

\footnotetext{
1 Una sociedad anónima abierta según nuestra Ley General de Sociedades Nº. 26887 del 1 de Enero de 1998, es aquella sociedad con un
} mínimo de 750 accionistas.

10/ QVIPUKAMAYOC|Vol. 23(43) 2015 
Tabla No 01:

Empresas que conforman la muestra del Estudio

\begin{tabular}{|c|c|c|r|}
\hline & RESUMEN & CANTIDAD & $(\%)$ \\
\hline 1 & Agroindustria & 14 & 14.0 \\
\hline 2 & Industriales & 45 & 45.0 \\
\hline 3 & Mineras & 15 & 15.0 \\
\hline 4 & Petroleras & 06 & 06.0 \\
\hline 5 & Servicio Público & 20 & 20.0 \\
\hline & TOTAL & 100 & 100.0 \\
\hline
\end{tabular}

Fuente: Elaboración propia.

La principal técnica utilizada en el estudio ha sido la Encuesta. El instrumento empleado se ha basado en un cuestionario de preguntas cerradas, las cuales permitieron identificar la situación actual a la problemática planteada en la presente investigación.

\section{MARCO TEÓRICO}

Los estados financieros representan el corazón del sistema de información empresarial y; por lo tanto, resulta fundamental para la toma de decisiones y el planeamiento estratégico de la organización. No obstante, en los últimos tiempos la contabilidad ha sufrido una evidente pérdida de fiabilidad y de relevancia social, fruto de la emergencia de un nuevo paradigma económico: la economía del conocimiento. Así, lo señalan diversos estudios (Di Piazza y Eccles; 2002), dando buena muestra de la pérdida de preeminencia de la información financiera al poner en evidencia cómo una parte significativa del valor, que los mercados atribuyen a las empresas se encuentra fuera de sus balances. Nos estamos refiriendo a los bienes de carácter intangible, que en una economía de mercado, adquieren una importancia decisiva para el buen funcionamiento y desarrollo de la empresa. Pues, no se ven reflejados en las hojas de los estados financieros como los otros activos: efectivo, inventarios o maquinarias; pero cada vez, tienen mayor valor contable.

Según Mesa-Velásquez (2012), "Los activos intangibles se pueden clasificar en identificables y no identificables. Para la norma internacional, son activos intangibles aquellos que fácilmente se pueden identificar, controlar y generar beneficios económicos a la empresa. Queda como tarea futura para la profesión contable, tratar de evaluar cómo se pueden medir y controlar los activos intangibles no identificables, ya que ellos también, le generan valor a una organización, pero la norma internacional no los acepta como activos intangibles".

En cambio, Fernández (2011), dice: "En los últimos tiempos conceptos como marca, reputación corporativa, responsabilidad social, capital intelectual o reputación interna acaparan el protagonismo en las principales tendencias dentro de la innovación en la gestión empresarial. No obstante, estos activos son realidad en la medida que se comunican ya que la comunicación empresarial es la interrelación estratégica de elementos, tales como la comunicación del producto, la comunicación de la empresa o corporativa, la gestión de los activos intangibles y la comunicación interna. La cultura y la gestión del conocimiento son activos gestionables, sobre todo, por la comunicación interna. La recopilación, transformación, transferencia, aplicación y protección del conocimiento deben ser administrados por medio de la comunicación y sus canales, desde dentro hacia fuera de la empresa".

Álvarez (2011), nos confirma su importancia: "La introducción de los activos intangibles en el balance empresarial no tiene lugar hasta los años 80 . Adam Smith, considerado el padre de la Economía moderna, pensaba que la esencia de la riqueza de una empresa era únicamente la producción de bienes materiales. Por lo que para él, el trabajo productivo creaba bienes tangibles que poseían valor en el mercado, mientras que sostenía la creación de intangibles como trabajo improductivo. Todo cambia cuando 
en 1912, Schumpeter sentencia en su Teoría de la Evolución Económica la idea de intangibilidad en el ámbito del sistema económico y la innovación y valora a los activos no tangibles como elementos fundamentales para el desarrollo y base de la empresa. A partir de ese momento, muchos investigadores han demostrado la importancia de los activos intangibles y su contribución a la generación de valor de una empresa, influyendo así tanto en su estructura interna como en la externa”.

Otro tema no menos importante pero significativo, son las nuevas actividades empresariales globales, herramientas fundamentales en la gestión de los negocios:

1) La planificación de la estrategia, instrumento que define la visión, misión y valores de la organización.

2) El cuadro de mando integral que favorece la transparencia en la gestión estableciendo un equilibrio entre las acciones inmediatas y las líneas estratégicas.

3) El capital intelectual con diferentes herramientas para medirlo como: El Método de la Q de Tobin; o el Valor Económico Agregado de Stern \& Shiely.
Que, a continuación desarrollaremos sucintamente para complementar nuestra orden de ideas.

\section{¿Qué es la Planificación Estratégica?}

No es intención de este artículo explicar el proceso de la planificación estratégica, sino informar de la importancia de uso.

Se puede señalar que, esta es una herramienta que ha sido creada en el mundo privado y que, dada su eficacia para enfrentar entornos dinámicos y fuertemente competitivos, ha ido adquiriendo una relevancia creciente en el sector público de tal magnitud que, actualmente, constituye uno de los ejes del proceso de Reforma y Modernización del Estado que se está impulsando en el Perú. Además, es sustancial tener siempre presente el carácter de herramienta que tiene la planificación y que este modelo deja de manifiesto, por cuanto entiende que su formulación e implementación no puede constituir un objetivo en sí mismo, como parecía ser en el caso de la planificación tradicional. La planificación no es más que un método como tantos otros, que pretende facilitar la consecución de los objetivos organizacionales a través de la entrega de una metodología”... (Aramayo, Orión, 2005).

\section{Gráfico No 01: Proceso de Planeamiento Estratégico}

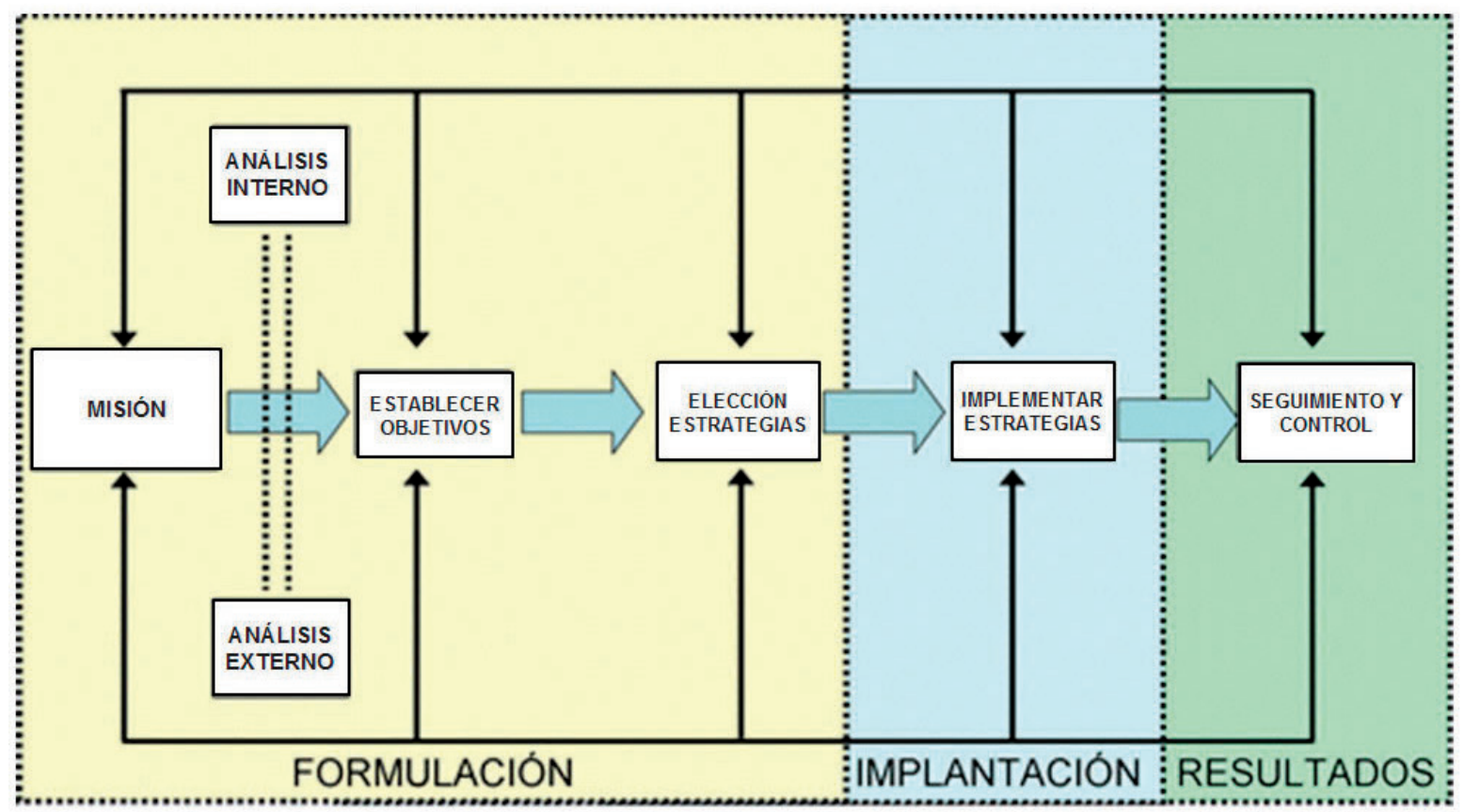

Fuente: Aramayo 2005

12/ QVIPURAMAYOC | Vol. 23(43) 2015 


\section{¿Qué es el Cuadro de Mando Integral? (CMI)}

Más conocido como Balance Scorecard (BSC), es una metodología que permite transmitir las estrategias definidas por una organización de manera clara y eficiente a todos los integrantes de la misma; y a la vez, poder traducir dichas estrategias en objetivos, acciones y medidas concretas que permitan saber si las mismas se están alcanzando.

Los creadores de este nuevo concepto de Gestión Estratégica son Robert Kaplan y David Norton, el cual ha sido expuesto en varios artículos de la Harvard Business Review. Básicamente plantean que el éxito de una Compañía es el resultado del encadenamiento equilibrado de las variables en cuatro perspectivas básicas: Finanzas, Clientes, Procesos Internos y, Aprendizaje y Crecimiento. (1996. The Balanced Scorecard. Translating Strategy into Action).

Las premisas fundamentales de este modelo son:

- Debe ayudarnos en nuestro proceso de definición de estrategias, objetivos, medidas, metas y acciones.

- Debe facilitarnos la comunicación de la dirección estratégica y ayudar a transmitir lo que debe hacer cada integrante de la organización para que sus acciones individuales aporten al cumplimiento de estos objetivos.

- Debe permitirnos comparar la evolución de las metas y su cumplimiento (real vs. planificado).

- Debe ser simple de entender y fácil de manejar para el usuario final y fácil de mantener para los administradores.

Como se puede observar, en la medida que las herramientas no estén integradas en un solo sistema informático, estas premisas se vuelven difíciles de cumplir y empiezan a transformarse en algunas de las razones para que la implementación de nuestro Cuadro de Mando Integral sea un fracaso.

\section{¿Qué es el Método de la Q de Tobin?}

Según la Enciclopedia de Economía ${ }^{2}$, es una relación por cociente entre el valor de mercado de la empresa y el coste de reemplazamiento de sus activos. Aquellas empresas cuya $Q$ es mayor que la unidad se sentirán estimuladas a invertir, puesto que el valor de mercado de la nueva inversión excederá a su coste; y viceversa, aquellas empresas cuya $Q$ sea inferior a la unidad se sentirán estimuladas a desinvertir. El proceso inversor (o desinversor) se detendrá cuando el valor de $Q$ sea igual a la unidad en todas las empresas de todos los sectores.

La Q de Tobin se utiliza también como indicador del poder competitivo o grado de monopolio de la empresa o del sector.

Se puede estimar dividiendo la suma del valor de mercado de las acciones de la sociedad más el valor de las deudas por el valor total de los activos de la sociedad según libros.

Un estudio realizado por economistas peruanos Montoro, C; y Navarro, A. (2010) que "estimar la Q de Tobin para la economía peruana mediante una serie de metodologías buscan corroborar empíricamente su validez para explicar la inversión agregada. Para ello, se realiza una revisión de diversas metodologías de estimación de la Q. Luego se utiliza información desagregada de la base de datos Economática entre los años 1999 y 2009, y se aplican cuatro enfoques diferentes para estudiar la robustez y la sensibilidad de las estimaciones. De esta manera, se calcula la $Q$ de Tobin utilizando la metodología de Chung y Pruitt (1994), la cual utiliza exclusivamente información contable, y se propone un híbrido de las metodologías empleadas por Perfect y Wiles (1994), y de Delgado y otros (2004). Además se estima una Q considerando únicamente las empresas que componen el Índice General de la Bolsa de Valores de Lima (IGBVL). Finalmente, se calcula una versión simple de la $Q$ de Tobin dada por el ratio de la capitalización bursátil respecto al valor total de activos de la empresa ${ }^{3}$.

2 http://www.economia48.com

3 Los interesados en el tema pueden visitar el trabajo de investigación denominado Estimación de la Q de Tobin para la economía peruana.(2010). Montoro, C; Navarro A. Banco Central de Reserva del Perú. Revista Estudios Económicos 19. Pág. 33-45. Lima. Perú. 


\section{¿Qué es el Valor Económico Agregado?}

O por sus siglas en inglés, Economic Value Added (EVA), se define según Ramírez (2005, pp 521-522) como "el remanente de operación que una empresa genera después de deducir a la utilidad de operación los impuestos y el cargo del costo de los recursos que son utilizados en la operación".

Asimismo, agrega las ventajas que el EVA brinda a las empresas que se indica a continuación:

1. Logra que los directores piensen, actúen y tomen decisiones como si fueran los dueños.

2. Promueve que se administre toda la organización con el enfoque del mejoramiento continuo.

3. Propicia que en la toma de decisiones siempre se considere el criterio que la opción óptima es aquella que genera el mayor valor, de ahí que se administre y se viva la cultura de la teoría del valor.

4. Crea la cultura de estar atentos a una buena administración de todos los activos.

5. Promueve la cultura de ser exigente y racionalizar los recursos ante los diferentes proyectos que se presentan.

6. Permite que al tomar decisiones se consideren tanto la operación como las estrategias, de tal manera que la meta de ambos sea la creación de valor.

7. Facilita una evaluación justa de la actuación de los directivos.

8. Promueve una constante preocupación por incrementar el valor de mercado del patrimonio de los accionistas.

Con respecto a los métodos de medición utilizados en las NIIF, se observa que este se debe adoptar, dependiendo de la naturaleza del activo y de la posibilidad que dicho cuente con un mercado activo. Por lo tanto, la aplicación de estos métodos queda sujeto a la interpretación de quién prepara la información, lo que en algunos casos puede resultar subjetivo.

Y así llegamos al fondo de la cuestión. Practicar la transparencia informativa tiene sus ventajas $y$ desventajas. Aquí, quizá ya estamos rozando con la información clave para la ventaja competitiva de la empresa. Dos extremos que identificamos en la definición de los intangibles futuros para la organización internacional. ¿Se superarán estas consideraciones? El mundo avanza a pasos acelerados. Solo el futuro lo dirá. Mientras tanto esperemos el pronunciamiento del IASB (Junta de Estándares Internacionales de Contabilidad), que se encuentra actualmente revisando el Marco Conceptual de las Normas Internacionales de Información Financiera que deben estar vigentes a partir del 1 de junio de 2015.

Un tema que se agrega a la discusión en el valor de los intangibles, es el caso de la nueva corriente denominada "Responsabilidad Social Empresarial”. A propósito veamos lo que nos dice el World Business Council for Sustainable Development 2000 "La Responsabilidad Social Empresarial es el compromiso continuo de la empresa de contribuir al desarrollo económico sostenible, mejorando la calidad de vida de sus empleados y sus familias, así como la de la comunidad local y de la sociedad en general". Porque se considera que son partes interesadas o grupos de interés del sector minero.

\section{Diferencia entre bienes intangibles y propiedad intelectual}

El tema que nos ocupa seguirá siendo motivo de discusión más en el ámbito tributario que filosófico. Así, Torres (2011), pone el punto sobre las íes:

"Es por ello, que se considera importante, en estas líneas, hacer referencia a qué son, qué tipos, sus características, las diferencias entre los mismos bienes intangibles, así como también, las diferencias que presentan con los bienes tangibles, puesto que, para los contribuyentes que poseen este tipo de activos, pueden representar grandes beneficios, pero a la vez, si no los manejan de manera adecuada, pueden representar serios problemas, mismos que van, desde los aspectos de protección de los activos, hasta las consecuencias fiscales que pueden derivarse de una indebida estrategia al momento de transmitirlos, cederlos o manifestarlos en el cumplimiento de sus obligaciones fiscales".

Así, en el Perú, estas diferencias de opinión entre el contribuyente y la Superintendencia Nacional de Administración Tributaria (SUNAT), han llegado a nivel del Tribunal Fiscal y más: el Tribunal Constitucional. Por consiguiente, es un tema delicado que debe definirse en algún momento de modo meridianamente claro. 


\section{Qué señala la NIC 38, Activos Intangibles}

Sus objetivos son los siguientes:

1) Establecer su tratamiento contable.

2) Determinar cuándo se reconoce un activo intangible.

3) Definir la medición del valor contable del activo intangible.

4) Qué revelaciones deben hacerse sobre activos intangibles.

Define que "Un activo intangible es un activo identificable, de carácter no monetario y sin apariencia física. Son activos monetarios tanto el dinero en efectivo como otros activos, por los que se van a recibir unas cantidades fijas o determinables de dinero. Surge por Investigación que es todo aquel estudio original y planificado, emprendido con la finalidad de obtener nuevos conocimientos científicos o tecnológicos".

Qué califican como Activos Intangibles:

- Marcas de fábrica.

- Membretes de diarios y nombres de publicaciones.

- Programas de computador (software).

- Licencias y franquicias.

- Derechos de autor, patentes y otros derechos de propiedad industrial, derechos de servicio y operación.

- Recetas, fórmulas, modelos, diseños, prototipos; y,

- Activos intangibles en desarrollo.

Qué No Califican como Activos Intangibles:

- Desembolsos para investigación.

- Egresos para desarrollo que no satisfacen condiciones de NIC 38.

- Salidas pre operativas.

- Partidas para capacitación.

- Gastos para publicidad y/o promoción.

- Descargos para reubicación o reorganización de la empresa.

Por tanto, se consideran como gasto.
Características fundamentales:

Factible identificación. Requiere ser perfectamente identificable para lo cual puede ser separable o no separable.

- Separable: La empresa puede alquilar, vender, cambiar o distribuir los beneficios económicos futuros atribuibles al citado activo. Ejemplo: Patentes, marcas de fábrica, derechos de autor.

- No separable: Aquellos que no pueden ser retirados de la entidad que los posea. Ejemplo: Plusvalía mercantil o goodwill.

Control sobre el activo. Cuando una empresa:

- Tiene el poder de obtener beneficios económicos que procedan de los recursos derivados del activo intangible.

- Puede restringir el acceso de terceros a tales beneficios económicos. Sustentado en derechos legales.

Ejemplo: Concesiones, patentes, marcas de fábrica debidamente registrados en el Instituto Nacional de Defensa de la Competencia y Protección de la Propiedad Intelectual (Indecopi).

Beneficios económicos futuros. Se pueden reconocer los beneficios económicos futuros que provengan de activos intangibles cuando puedan generar ingresos por venta de bienes o servicios o ahorros de costos. Ejemplo: Fórmulas secretas (Know how).

Costo de confiable valuación. Se puede determinar el importe de efectivo o su equivalente de efectivo a pagar o el valor razonable de la contraprestación entregada para obtener el activo. Ejemplo: Importe pagado por una franquicia según contrato suscrito.

\section{Qué señala la NIIF 6, Exploración y Evaluación de Recursos Naturales}

Específica la información financiera sobre desembolsos incurridos en la exploración y evaluación de recursos minerales antes de que se pueda demostrar la factibilidad técnica y viabilidad comercial de la extracción de los recursos minerales. No explica la información financiera para el desarrollo de los recursos minerales. 
Los desembolsos de exploración y evaluación $y$, los derechos minerales se excluyen del alcance de las normas que tratan sobre los activos intangibles y propiedades, planta y equipo. Norma emitida como una medida a corto plazo para rellenar una laguna en las NIIF. En ausencia de la NIIF 6, se requeriría a las entidades contabilizar los desembolsos de exploración y evaluación de acuerdo con las normas que tratan sobre elementos similares, y las definiciones, criterios de reconocimiento y conceptos de medición para activos y gastos del Marco Conceptual para la Preparación y Presentación de Estados Financieros. Para la mayoría de las entidades, la aplicación de otras normas y del Marco Conceptual habría dado lugar a cambios contables.

En muchos sentidos, una entidad puede continuar contabilizando los desembolsos de exploración y evaluación utilizando las mismas políticas contables que aplicaba inmediatamente antes de adoptar la NIIF. Sin embargo, la norma lleva a cabo algunas mejoras limitadas para contabilizar estos desembolsos:

- La entidad debe determinar las políticas contables especificando qué desembolsos de exploración y evaluación se reconocen como activos, y cómo se miden estos activos.

- En el reconocimiento, los activos de exploración y evaluación se miden al costo. Posteriormente, se evalúan utilizando el modelo del costo o el de revaluación.

- Los activos de exploración y evaluación se clasifican como activos tangibles o intangibles de acuerdo con su naturaleza.

- Un activo de exploración y evaluación se evalúa por deterioro de valor.

\section{Servicio Interno de Ingresos de los Estados Unidos de Norte América (IRS, Internal Revenue Service)}

Equivalente a nuestra SUNAT (Superintendencia Nacional de Aduanas y Administración Tributaria) peruana, al referirse a los activos intangibles, los describe como activos cuyo valor deriva no de sus cualidades físicas sino de su contenido intelectual, clasificándolas en seis categorías: a. Tecnología: Patentes, inventos, fórmulas, procesos, diseños, modelos, know-how.

b.Literatura: Derechos de autor, obras literarias, musicales o artísticas.

c. Ventas: Marcas comerciales, razones comerciales.

d. Organizaciones Empresariales: Franquicias, licencias o contratos.

e. Operaciones y listas: Métodos, Programas, Sistemas, Procedimientos, Campañas, Estudios, Pronósticos, Estimaciones, listas de clientes o información técnica.

f. Otros Artículos similares: Cuyo valor derive de su contenido intelectual más que de sus atributos físicos.

\section{Organización para la Cooperación y Desarrollo Económicos (OCDE)}

Este organismo internacional se fundó en 1961, y en la actualidad, se encuentra integrado por 33 países miembros comprometidos con una economía de mercado abierta, democracia pluralista y respeto a los derechos humanos; en el capítulo VI al referirse a los bienes intangibles lo hace como aquellos derechos que se derivan de la capacidad intelectual humana, incluyendo inventos, trabajos escritos, diseños industriales y trabajos artísticos.

Como podemos observar, todas las definiciones coinciden en que los activos intangibles son:

- Recursos que posee la empresa.

-Inmateriales; es decir, no pueden ser percibidos por los sentidos.

Los activos intangibles no se perciben pero se conoce de su existencia y de su aporte a la generación de valor para las empresas. Asimismo, representan derechos o privilegios que tienen la particularidad de permitir la reducción de los costos de producción, de mejorar la calidad de un producto o de promover su aceptación en un mercado determinado.

\section{INTERPRETACIÓN DE LOS DATOS}

Ha sido necesario preparar un pequeño cuestionario de tres preguntas, el cual ha sido aplicado a la muestra de 100 empresas que listan en la Bolsa de 
Valores de Lima (BVL), al 31 de diciembre de 2014, dirigida a los Contadores, las cuales han sido elegidas por su volumen de ventas y por su nivel de negocios internacionales, cuyos resultados fueron los siguientes:

Tabla No 02:

Su empresa: ¿Cuenta con activos intangibles?

\begin{tabular}{|c|c|c|c|c|c|c|c|}
\hline & \multicolumn{5}{|c|}{ EMPRESAS ENCUESTADAS } & \multicolumn{2}{c|}{ TOTAL ENCUESTAS } \\
\hline RPTA. & AGRO-IND. & INDUSTRIA & MINERAS & PETRÓLEO & SERV. PÚB & RPTA. & $(\%)$ \\
\hline $\mathrm{Si}$ & 14 & 45 & 15 & 6 & 20 & 100 & 100 \\
\hline $\mathrm{No}$ & 0 & 0 & 0 & 0 & 0 & 0 & 0 \\
\hline No opina & 0 & 0 & 0 & 0 & 0 & 0 & 0 \\
\hline TOTAL & $\mathbf{1 4}$ & $\mathbf{4 5}$ & $\mathbf{1 5}$ & $\mathbf{6}$ & $\mathbf{2 0}$ & $\mathbf{1 0 0}$ & $\mathbf{1 0 0}$ \\
\hline
\end{tabular}

Fuente: Elaboración propia.

El 100\% de los encuestados contestaron que sí. Todas las empresas en mayor o menor grado cuentan con activos intangibles. Se confirma así, la importancia de este tipo de inversiones que tienen las organizaciones en el interior de sus activos.

Tabla No 03:

¿Aplica su empresa un plan estratégico de largo plazo?

\begin{tabular}{|c|c|c|c|c|c|c|c|}
\hline & \multicolumn{5}{|c|}{ EMPRESAS ENCUESTADAS } & \multicolumn{2}{c|}{ TOTAL ENCUESTAS } \\
\hline RPTA. & AGRO-IND. & INDUSTRIA & MINERAS & PETRÓLEO & SERV. PÚB & RPTA. & $(\%)$ \\
\hline $\mathrm{Si}$ & 14 & 45 & 15 & 6 & 20 & 100 & 100 \\
\hline $\mathrm{No}$ & 0 & 0 & 0 & 0 & 0 & 0 & 0 \\
\hline No opina & 0 & 0 & 0 & 0 & 0 & 0 & 0 \\
\hline TOTAL & $\mathbf{1 4}$ & $\mathbf{4 5}$ & $\mathbf{1 5}$ & $\mathbf{6}$ & $\mathbf{2 0}$ & $\mathbf{1 0 0}$ & $\mathbf{1 0 0}$ \\
\hline
\end{tabular}

Fuente: Elaboración propia.

El 100\% de los encuestados contestaron que sí. Se confirma así, la importancia que dan las empresas que listan en la BVL, a la prospección del futuro. Pero muy pocos avizoraron la caída de la economía en el año corriente, como se viene confirmando por informaciones periodísticas, conforme avanza el año 2015.

Tabla No 04:

¿Es necesario ampliar el marco conceptual en la parte no financiera relacionada con los activos intangibles?

\begin{tabular}{|c|c|c|c|c|c|c|c|}
\hline & \multicolumn{4}{|c|}{ EMPRESAS ENCUESTADAS } & \multicolumn{2}{c|}{ TOTAL ENCUESTAS } \\
\hline RPTA. & AGRO-IND. & INDUSTRIA & MINERAS & PETRÓLEO & SERV. PÚB & RPTA. & $(\%)$ \\
\hline $\mathrm{Si}$ & 10 & 32 & 10 & 4 & 14 & 70 & 70 \\
\hline $\mathrm{No}$ & 0 & 0 & 0 & 0 & 0 & 0 & 0 \\
\hline No opina & 4 & 13 & 5 & 2 & 6 & 30 & 30 \\
\hline TOTAL & $\mathbf{1 4}$ & $\mathbf{4 5}$ & $\mathbf{1 5}$ & $\mathbf{6}$ & $\mathbf{2 0}$ & $\mathbf{1 0 0}$ & $\mathbf{1 0 0}$ \\
\hline
\end{tabular}

Fuente: Elaboración propia. 
El 70\% de los encuestados contestaron que sí es necesario y el $30 \%$ no opinó. Los Contadores Públicos encuestados conocen el contenido del marco conceptual de la norma internacional, pero aún hay un sector que se abstiene por la complejidad de su valorización.

\section{Resultados obtenidos}

El hallazgo principal del estudio, nos muestra en función de lo expuesto en acápites anteriores, que sería necesario acordar un marco conceptual más amplio que abordara la problemática integral de la información financiera y no financiera, restringida exclusivamente a lo tradicional.

La NIIF 6, Exploración y Evaluación de Recursos Naturales, no explica la información financiera para el desarrollo de los recursos minerales, y en este aspecto, en el Perú hay serios problemas en temas del medio ambiente y conflictos sociales con los grupos de interés, como son las comunidades propietarias de la superficie en lugares del país donde se ha detectado yacimientos mineros de oro $y / o$ cobre.

Nuestra Administración Tributaria no acepta los gastos efectuados por las empresas mineras a favor de la comunidades ubicadas en el área de influencia de su yacimiento minero, llámese escuelas, postas médicas, etc., pues considera que constituyen actos de liberalidad no deducibles para efectos de la determinación del Impuesto a la Renta (IR). Pero los contribuyentes sí consideran que estos conceptos constituyen gastos deducibles pues son necesarios para el normal desarrollo de sus actividades productivas y han sido otorgadas en cumplimiento de obligaciones asumidas en los convenios celebrados con las comunidades de la zona de influencia de la concesión. Que constituye un derecho distinto, separado e independiente de los derechos sobre el predio donde se encuentre ubicada; es decir, no otorga derechos sobre la superficie. $\mathrm{Y}$ en este sentido, el artículo $\mathrm{N}^{\circ} 66$ de la Constitución Política peruana, señala que los recursos naturales son patrimonio de la Nación, siendo la concesión el mecanismo que permite su explotación y que confiere el respectivo derecho real.

En efecto, el Ministerio de Energía y Minas, mediante el D.S. $\mathrm{N}^{\circ}$. 042-2003-EM incorporó como un requisito para obtener concesiones mineras la obligación de presentar un compromiso previo, en forma de declaración jurada, mediante la cual el solicitante se obligaba, entre otros, a colaborar con las poblaciones del área de influencia de la mina, en la creación de oportunidades de desarrollo. Actuando en consecuencia, 10 años después el Tribunal Fiscal en Resolución Nº. 18397-10-2013 ha resuelto "que los gastos indicados no constituyen donaciones o liberalidades; es decir, actos de desprendimiento sin esperar recompensa, sino que surgen en virtud de una obligación asumida con motivo del otorgamiento de la concesión”. Reconoce que los gastos efectuados por concepto de responsabilidad social constituyen instrumentos legítimos utilizados por las empresas para la generación de ingresos; así como, para el mantenimiento de su fuente productora, y se realizan en cumplimiento del compromiso asumido por el concesionario minero con el Estado, por lo que cumplen con el principio de causalidad y son deducibles para efecto de la determinación del impuesto a la renta.

Reconocimiento acertado por parte de la autoridad nacional que hace prever a futuro, la solución armoniosa de los conflictos sociales, en el sector minero, entre la empresa inversora y las comunidades del entorno.

Las debilidades de las NIIF se presentan en aquellos activos no considerados como intangibles:

\section{a) En el Factor Humano:}

El Know-how.

El personal formado que figura en la planilla de la empresa.

Fidelidad hacia la organización.

La motivación como motor de sinergia.

\section{b) En el Factor Cliente:}

Fidelidad del cliente hacia los productos que distribuye la empresa.

Canales de distribución empleados por la organización.

Base de datos de los clientes.

Asociaciones de Clientes.

c) En el Factor Organizacional:

Sistemas de información.

Filosofía de la gestión. 
Cultura empresarial.

Procesos de gestión.

\section{Guía de Discusión}

1. ¿Qué necesitamos demostrar a la autoridad tributaria para hacer valer como intangibles al Capital Intelectual integrado por el Capital Humano, el Capital Estructural y el Capital Relacional?

2. ¿El IASB ampliará el Marco Conceptual de las Normas Internacionales de Información Financiera validando las nuevas herramientas de gestión?

3. ¿Están las empresas dispuestas a practicar el Principio de Transparencia hasta el límite de hacer conocer al mercado el secreto de su ventaja competitiva?

\section{CONCLUSIONES}

1. Instituciones internacionales como el Servicio Interno de Ingresos de los Estados Unidos de Norteamérica (IRS) y la Organización para la Cooperación y Desarrollo Económicos (OCDE), coinciden en identificar qué es un bien intangible según lo especifica la NIC 38. Pero no hay evidencias claras por parte del IASB, para reconocer como intangibles a las herramientas fundamentales en la gestión de los negocios que utilizan las empresas de clase mundial integrados en el Factor Humano, el Factor Cliente y el Factor Organizacional.

2. Es de necesidad pública ampliar el Marco Conceptual para la Preparación y Presentación de Estados Financieros de las normas internacionales vigentes, no solo a la información financiera, sino también, a la no financiera. Así, podrán validarse instrumentos prospectivos como la Planificación Estratégica, el Cuadro de Mando Integral, la Q de Tobin o el Valor Económico Agregado. Que hacen que la ciencia contable sea predictible en el largo plazo.

3. Si la controversia se plantea en los términos antes esbozado, solo quedaría promocionar el uso obligatorio de las herramientas de gestión ya citados. De paso realzamos y normalizamos el nivel técnico de la información no financiera a nivel global.

\section{RECOMENDACIÓN}

1. Para colaborar en la difusión de la normatividad del IASB, además de hacer de conocimiento de la comunidad empresarial mundial el nuevo Marco Conceptual para la Preparación y Presentación de Estados Financieros, se exhorta a las universidades públicas y privadas del país, que cuentan con facultades de Ciencias Empresariales, la inclusión preferentemente en sus syllabus de pre y posgrado, cursos dedicados a la enseñanza y capacitación en temas como: Planeamiento Estratégico, Cuadro de Mando Integral, la Q de Tobin y/o el Valor Económico Agregado con casuísticas aplicadas a la empresa nacional y/o internacional.

\section{REFERENCIA BIBLIOGRÁFICA}

1. ARAMAYO, O. (2005) Manual de Planificación Estratégica. Universidad de Chile. Instituto de la comunicación e imagen.

2. ÁlVAREZ, C. (2011) Hacia un Nuevo Modelo de Valoración de Intangibles. Inglaterra.

3. BOLSA DE VALORES DE LIMA (2014) Estados Financieros Comparados. Diciembre 2014. Lima. Perú.

4. CAÑIBANO, L., GARCÍA-AYUSO, MY SÁNCHEZ, P. (2000) "Accounting for Intangibles: A Literature Review" .Journal of Accounting Literature, 19:102-130.

5. CONSEJO MUNDIAL DE REUNIONES LABORALES PARA EL DESARROLLO SOSTENIBLE 2000 (WBCFSD 2000) Consejo Empresarial Mundial para el Desarrollo Sostenible. Véase la página Web: www.wbcsd.org

6. CHUNG, K. y PRUITT S. A. Simple Approximation of Tobin's Q. Revista Financial Management, volumen 23, número 3, 1994.

7. DI PIAZZA, S.A. y ECCLES, R.G. (2002) Recuperar la confianza: el futuro de la información corporativa. FT Prentice-Hall. Madrid.

8. FERNÁNDEZ, C. (2011) Potenciar los Activos Intangibles en el Capital Humano, Innovando el Plan Estratégico de Comunicación Interna. Universidad de Valladolid. España. 
9. IASB (2004) NIC 38. Activos Intangibles.

10. IASB (2012) NIIF 6. Exploración y Evaluación de Recursos Minerales.

11. KAPLAN R.S. y D. NORTON (1996). “The Balanced Scorecard" Harvard Business School Press.

12. KAPLAN R.S. y D. NORTON (1992) "The Balanced Scorecard - Measures that drive performance". Harvard Business Review (HBR).

13. LAGRANENCICLOPEDIADEECONOMIA. http://www.economia48.com.

14. MESA-VELÁSQUEZ, G. (2012) Medición de los activos intangibles, retos y desafíos. Cuadernos de Contabilidad. Bogotá. Colombia.

15. MINISTERIO DE ENERGÍA Y MINAS (2003) Decreto Supremo No 042-2003-EM- establece compromiso previo como requisito para el desarrollo de actividades mineras y normas complementarias. Lima. Perú.

16. MONTORO, C. NAVARRO, A. (2010) Estimación de la Q de Tobin para la economía peruana. Banco Central de Reserva del Perú. Revista Estudios Económicos. Pág. 33-45. Lima. Perú.

17. OCDE. ORGANIZACIÓN PARA LA COOPERACIÓN Y DESARROLLO ECONÓMICOS (2003) Artículo 12 del Convenio Modelo OCDE para evitar la doble imposición. Véase la página Web: www.oecd.org.
18. PERFECT, S.B.y WILES, K.W. Alternative Constructions of Tobin's Q: An Empirical Comparison. Journal of Empirical Finance.

19. RAMÍREZ, D.(2005) Contabilidad Administrativa. McGraw-Hill /INTERAMERICANA EDITORES, S.A. DE C.V. México D.F. México

20. SÁNCHEZ, T. (2004) Estudio Comparativo de los Convenios Suscritos por España Respecto al Convenio Modelo de la OCDE. Subdirección General de Tributación de no Residentes. Madrid. España.

21. SCHUMPETER, J. (1978) Teoría del Desenvolvimiento Económico. Quinta Reimpresión. Fondo de Cultura Económica, México.

22. TORRES, V. (2011) Los Activos Intangibles en la Empresa. Revista Académica de Investigación “Tlatemoani”. No. 5. México D.F. México.

23. TRIBUNAL FISCAL (2013) Resolución No. 18397-10-2013. Gastos efectuados por concepto de responsabilidad social, constituyen instrumentos legítimos para el mantenimiento de su fuente productora. Lima. Perú. Véase la página Web: www.mef.gob.pe

24. TYLES, M. (2006) Los Activos Intangibles: limitaciones en la contabilidad financiera y oportunidades en la contabilidad de gestión. 